\title{
Search for magnetic monopoles with nuclear track detectors
}

\author{
M. Giorgini ${ }^{\mathrm{a}}$ for the MACRO Collaboration* \\ ${ }^{a}$ Dipartimento di Fisica, Università di Bologna and INFN, Sezione di Bologna \\ Viale C. Berti Pichat 6/2, 40127 Bologna, Italy \\ e-mail: miriam.giorgini@bo.infn.it
}

\begin{abstract}
This paper describes an experimental search for GUT magnetic monopoles in the MACRO experiment using the nuclear track subdetector CR39. After discussing the working principle, the charge resolution and the calibration of the detector, the experimental procedure for searching for magnetic monopoles is described. Since no candidates were found, the upper flux limits obtained by the MACRO CR39 used as a "stand alone detector" for magnetic monopoles of different magnetic charges are presented.
\end{abstract}

\section{INTRODUCTION}

Massive magnetic monopoles $\left(M>10^{16} \mathrm{GeV}\right)$ are predicted by all Grand Unified Theories of the electroweak and strong interactions (GUTs) 11. They could carry a magnetic charge $g=n g_{D}$, where $n$ is an integer and $g_{D}$ is the elementary magnetic charge predicted by Dirac [2]. They should have been produced very early in the Big Bang Universe and should have cooled to very low velocities since then.

MACRO (Monopole, Astrophysics and Cosmic Ray Observatory) [3] is a large area underground experiment operating at Laboratori Nazionali del Gran Sasso, Italy, at an average depth of 3700 $\mathrm{hg} / \mathrm{cm}^{2}$. It has a modular structure and three types of subdetectors: streamer tubes, liquid scintillators and nuclear track detectors (CR39 and Lexan).

The track-etch system is distribuited on three ortogonal planes for a total area of $1263 \mathrm{~m}^{2}$.

In this paper the calibration, the charge resolution and the results of the magnetic monopole (MM) search using the MACRO CR39 as a "stand alone detector" are discussed. In particular, the attention is focused on the experimental procedure of the search for magnetic monopoles: the etching conditions and the methods of searching for candidate events. In absence of candidates, an updated estimate of the upper limits for an isotropic flux of monopoles

\footnotetext{
*For the complete list of the Collaboration see the paper by L. Patrizii in these Proceedings.
}

with magnetic charge $g=g_{D}, 2 g_{D}, 3 g_{D}$ and for monopole-proton $(\mathrm{M}+\mathrm{p})$ composites established by the MACRO nuclear track detector is also presented.

\section{THE CR39 NUCLEAR TRACK DE- TECTOR}

The MACRO CR39 is manufactured by the Intercast Europe Company of Parma (Italy). The standard CR39, used mainly for sun glasses, was improved in order to achieve a lower detection threshold, a higher sensitivity in a large range of energy losses, a high quality of the post-etched surfaces after prolonged etching, stability of the sensitivity of the polymer over long periods of time and uniformity of sensitivity [4].

\subsection{Working principle}

When a heavily ionizing particle crosses a nuclear track detector foil, it produces damages at the level of molecular bonds, forming the so called "latent track". During the chemical etching of the detector in a basic water solution, as the etching velocity along the "latent track", $v_{T}$, is larger than that for the bulk material, $v_{B}$, etch-pit cones are formed on both sides of the foil, see Fig. 1. The base area and the height of each cone are functions of the Restricted Energy Loss (REL) of the incident ion and thus of the charge $Z$ [5,6].

\subsection{Charge resolution of the CR39 detec- tor \\ Stacks of CR39-target-CR39 were exposed to a $\mathrm{Pb}^{82+}$ ion beam of $158 \mathrm{~A} \mathrm{GeV}$ at the CERN-}


2

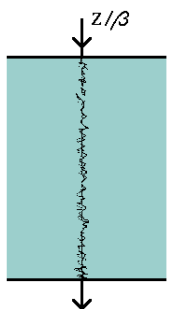

(a)

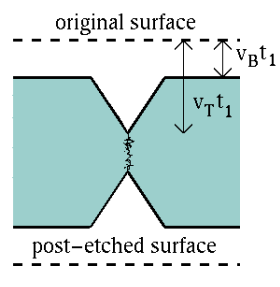

(b)

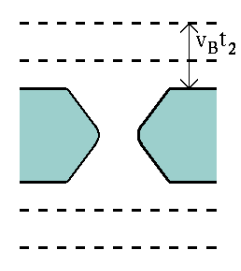

(c)
Figure 1. (a) In a polymeric nuclear track detector, a heavily ionizing particle breaks the polymeric bonds along its path, leaving reactive molecules; (b) a chemical etching in a basic water solution acts globally with velocity $v_{B}$ and preferentially along the path of the particle with velocity $v_{T}$, forming, after the time $t_{1}$, observable cones on the post-etched surfaces and, after $t_{2}$, a hole (c).

SPS. The exposures were performed at normal incidence. The beam passed through some foils of CR39 detector, interacted in the target material and then passed through CR39 foils which recorded the surviving lead projectiles and their fragments. After exposure, the CR39 detectors were etched for $268 \mathrm{~h}$ in a $6 \mathrm{~N} \mathrm{NaOH}$ water solution at a temperature of $45^{\circ} \mathrm{C}$.

For each etch-pit cone, the base area was measured with an automatic image analyzer system. In order to reconstruct the path of the ions, we used a tracking procedure and then we performed for each ion an average of the measured areas. A distribution of etched cone base areas averaged on $12 \div 14$ faces of the CR39 sheets located after the fragmentation target is shown in Fig. 2. This procedure has a good charge resolution for $Z \leq 60 e$ and an acceptable one up to $Z \sim 74 e$; for $Z \geq 75 e$ the fragment tracks and the lead tracks cannot be distinguished. The charge resolution at $Z \sim 10 e$ is $\sim 0.3 e$ for a single measurement and $\sim 0.09 e$ for the average of 12 measurements [7]. The charge resolution is worsening with increasing charge of the ions.

For high $Z$ nuclei, the height of the etched cone is more sensitive to $Z$ than its base area or diameter [8]. In order to separate the surviving lead ions from the nuclear fragments with charge $Z \geq$ $75 e$, we performed manual cone height measurements with an optical microscope. The charge resolution obtained from this measurement technique is about $0.19 e$ on a single face of the detec-

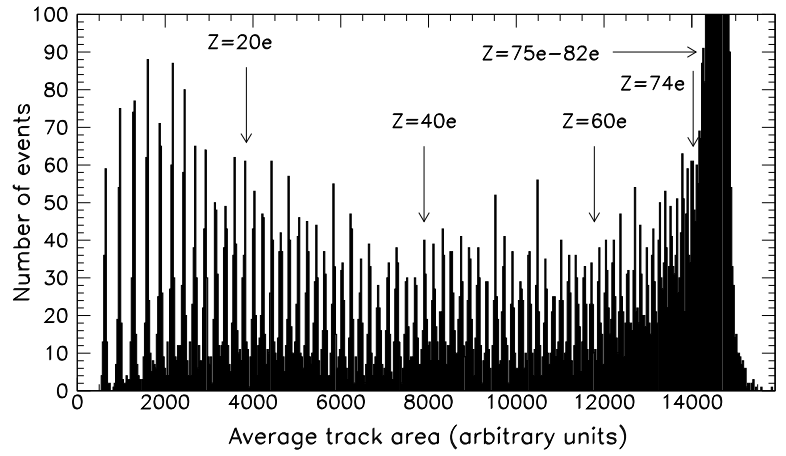

Figure 2. Distribution of etched cone base areas averaged on $12 \div 14$ faces of the CR39 sheets located after the fragmentation target. This procedure allows to separate all single peaks up to $Z=74 e$; for $Z \geq 75$ e the fragment tracks and the lead tracks cannot be distinguished.

tor, see Fig. 3 .

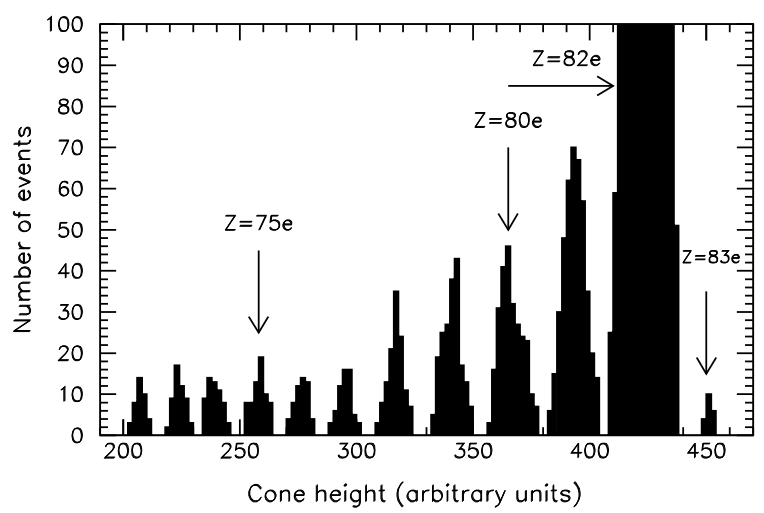

Figure 3. Distribution of etched cone heights in a CR39 sheet located after the fragmentation target.

\subsection{Calibration of CR39 detectors}

This work was aimed at establishing the dependence of the response of the CR39 nuclear track detector on the energy losses of charged particles. The calibration curve of a nuclear track detector is the plot of the reduced etch rate, $p=v_{T} / v_{B}$, versus REL. For an ion passing through a nuclear track detector, REL is the fraction of the energy loss localized in a cilindrical region with diameter of $100 \AA$ around its path. It is the energy loss which leads to $\delta$ rays with energies lower than $E_{\max }$, where $E_{\max } \simeq 200 \mathrm{eV}$ for CR39.

After the exposure described in $\S 2.2$, using 
measurements of the post-etched cone base areas and heights in the charge regions $7 e \leq Z \leq 74 e$ and $75 e<Z \leq 83 e$, respectively, we obtained the calibration curve shown in Fig. 4 .

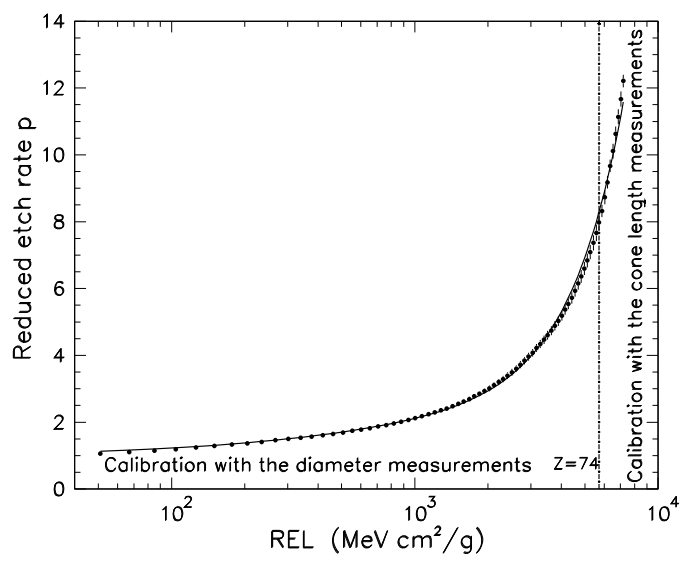

Figure 4. Reduced etch rate $p$ vs REL for the MACRO CR39 exposed to a relativistic lead beam; the points are the experimental data, the solid line is the best fit to the data points.

The comparison of this result with previous calibrations using relativistic and low velocity ions [6] shows that a unique curve describes, within errors, all the data. We can conclude that the response of the detector depends only from REL.

\section{THE MACRO TRACK-ETCH SYS- TEM}

The MACRO track-etch detector consists of two types of nuclear track detectors, CR39 and Lexan. Most of the work concerns the development and processing of the CR39. Lexan has a much higher threshold compared to that of CR39, making it sensitive to relativistic monopoles only. Lexan has not been used until now for monopole searches in MACRO.

The MACRO track-etch detector is organized in stacks ("wagons") consisting of three layers of CR39, each about $1.4 \mathrm{~mm}$ thick, three layers of Lexan, each $0.25 \mathrm{~mm}$ thick, and an aluminium absorber $1 \mathrm{~mm}$ thick, Fig. 5 . Each stack has a surface of $24.5 \times 24.5 \mathrm{~cm}^{2}$ and is placed in an aluminized mylar bag filled with dry air.

The main purpose of the track-etch detector is to offer independent measurements of MMs. It

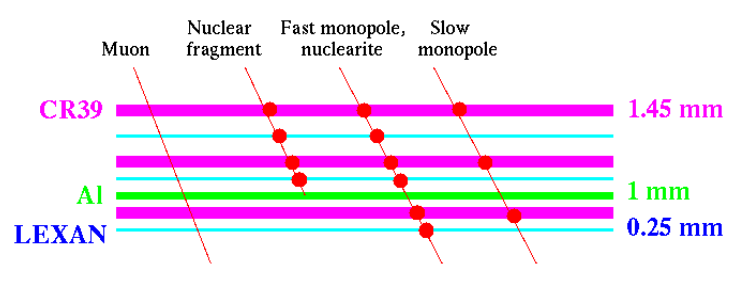

Figure 5. Stack of nuclear track detectors used in MACRO. The black points indicate the breaking of polymeric bonds forming the "latent track".

may be used both as a "stand alone detector" and "triggered" by other MACRO subdetectors.

\subsection{The response of CR39 to monopoles}

The Restricted Energy Loss as a function of the monopole velocity in CR39 for $g=$ $g_{D}, 2 g_{D}, 3 g_{D}, 6 g_{D}, 9 g_{D}$ bare monopoles is shown in Fig. 6 [9]. The horizontal dashed line represents the detection threshold for the MACRO CR39, corresponding to the REL value of $\sim 26 \mathrm{MeV} \mathrm{cm}^{2} \mathrm{~g}^{-1}$ and to $Z / \beta \sim 5$.

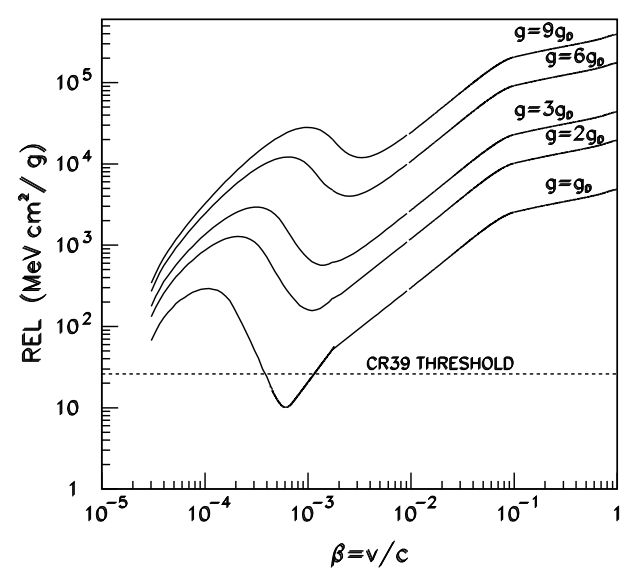

Figure 6. REL vs $\beta$ for magnetic monopoles of different magnetic charge $g$.

The detection of MMs is limited to poles with incidence angles smaller than a critical value $\delta_{c}$ with respect to the normal; one has $\delta_{c}=$ $\arccos \left(p^{-1}\right)$, where $p=v_{T} / v_{B}$ [5].

In order to determine the acceptance of the MACRO CR39, we performed a Monte Carlo simulation, assuming an isotropic flux of monopoles. This requires that the masses of the monopoles should be greater than $10^{17} \mathrm{GeV}$ and their veloc- 
ities should be larger than $\beta=3 \times 10^{-5}$ for $g=g_{D}$ monopoles and $\beta=5 \times 10^{-5}$ for $g=2 g_{D}, 3 g_{D}$ monopoles and $(\mathrm{M}+\mathrm{p})$ aggregates. The total acceptance of the MACRO CR39 subdetector is $7100 \mathrm{~m}^{2} \mathrm{sr}$ for an isotropic flux of fast monopoles.

\subsection{Experimental procedure and results}

The passage of a MM in CR39 is expected to cause a structural damage. Chemical etching should result in collinear etch-pit cones of equal size on both faces of each sheet. After extraction from the MACRO apparatus, three fiducial holes are drilled in each wagon by a precision drilling machine. The next steps are: (i) a "strong" etching in $8 \mathrm{~N} \mathrm{NaOH}$ water solution at $85{ }^{\circ} \mathrm{C}$ for 50 hours is applied to the first upper sheet of each wagon; (ii) after etching, we perform a first scan in transparency and a successive analysis with a binocular microscope with low magnification $(16 \times)$, looking for holes and/or corresponding double etch-pits; (iii) in case of a candidate in the upper sheet, a "standard" etching in $6 \mathrm{~N} \mathrm{NaOH}$ water solution at $70{ }^{\circ} \mathrm{C}$ for 30 hours is applied to the third sheet. A good candidate track must satisfy a three-fold coincidence of the position, incident angles among the layers and should also give the same value of REL.

From 1991 until now we etched $227 \mathrm{~m}^{2}$ of CR39, with an average exposure time of 7.6 years. Since no candidates were found, the $90 \%$ C.L. upper limits are given by $\Phi<2.3 /(S \Omega \cdot \Delta t)$ where $S \Omega$ is the acceptance of the extracted wagons and $\Delta t$ is their exposure time. Since the limits corresponding to each individual extraction are independent, the resulting MACRO CR39 limit is given by the sum of each individual $(S \Omega \cdot \Delta t)$ contribution. In Fig. 7 we present the flux upper limits for $g=g_{D}, 2 g_{D}, 3 g_{D}$ bare MMs and for $\mathrm{M}+\mathrm{p}$ composites obtained by our analysis.

\subsection{Byproducts of the search for MMs}

The searches for magnetic monopoles based on the nuclear track detectors may also be applied to search for other rare particles.

Nuclearites are hypothesized nuggets of strange quark matter and possible candidates for the dark matter. The CR39 detector may detect nuclearites with $\beta>10^{-5}$. In absence of candidates,

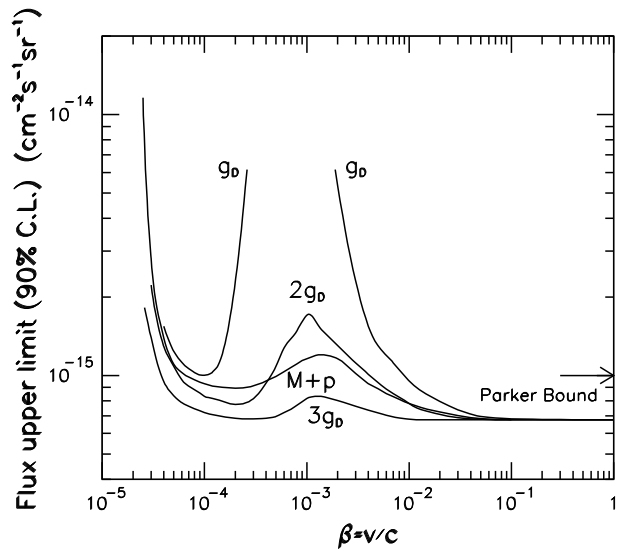

Figure 7. $90 \%$ C.L. upper limits for magnetic monopoles obtained using MACRO CR39 as a "stand alone detector".

the $90 \%$ C.L. upper limit for an isotropic flux of nuclearites with $\beta>10^{-5}$ is discussed in refs. [10].

Q-balls should be supersymmetric coherent states of squarks, sleptons and Higgs fields. The CR39 detector may detect charged Q-balls with $10^{-4}<\beta<10^{-2}$.

\section{REFERENCES}

1. G. t'Hooft, Nucl. Phys. B79 (1974) 276.

2. P. A. M. Dirac, Proceedings of the Royal Society, London, A133 (1931) 60.

3. S. Ahlen et al., MACRO Coll., Nucl. Instr. and Meth. in Phys. Res. A324 (1993) 337.

4. S. Ahlen et al., MACRO Coll., Nucl. Tracks Radiat. Meas. 19 (1991) 641.

5. R. Fleischer, P. B. Price and R. M. Walker, Nuclear Tracks in Solids, University of California Press (1975).

6. S. Cecchini et al., Nuovo Cim. A109 (1996) 1119.

7. H. Dekhissi et al., CERN Prep. EXT-98-010, to be published in Nucl. Phys. A.

8. G. Giacomelli et al., Nucl. Instr. Meth. A411 (1998) 41.

9. J. Derkaoui et al., Astrop. Phys. 10 (1999) 339.

10. M. Ambrosio et al., MACRO Coll., hepex/9904031, submitted to Eur. Phys. Jou. C; L. Patrizii for the MACRO Coll. in these Proceedings (1999). 